\title{
$A b$ initio energetics for modeling phase stability of the Np-U system
}

Wei Xie ${ }^{1}$, Y. Austin Chang ${ }^{1,2, a}$, Dane Morgan ${ }^{1,2,3, b}$

${ }^{1}$ Materials Science Program, University of Wisconsin-Madison, Madison WI 53706, United States

${ }^{2}$ Department of Materials Science and Engineering, University of Wisconsin-Madison, Madison WI 53706, United States

${ }^{3}$ Department of Engineering Physics, University of Wisconsin-Madison, Madison WI 53706, United States

${ }^{\mathrm{a}}$ Deceased.

${ }^{\mathrm{b}}$ Corresponding author. Address: 1509 University Avenue, Madison WI 53706, United States. Tel.: + 1608265 5879. Fax: +1 608262 8353. Email address: ddmorgan@wisc.edu. 


\begin{abstract}
The Np-U system has been rarely studied despite this alloy being one key binary subsystem of metallic nuclear fuels. This study aims to further the understanding of the phase stability of the Np-U system through $a b$ initio calculations. We first examined an existing CALPHAD model based on available experimental phase boundary data. We then focused on phases of which the CALPHAD model reproduces reasonably the experiment data and examined if values of the effective Hubbard $U$ (i.e., $U_{\text {eff }}$ ) parameter for $\mathrm{Np}$ and $\mathrm{U}$ fitted previously in the $\mathrm{Np}-\mathrm{Zr}$ and $\mathrm{U}-\mathrm{Zr}$ systems are also applicable to the $\mathrm{Np}-\mathrm{U}$ system, using CALPHAD predicted enthalpies as references. We found that in general DFT $+U$ predicts improved or at least similarly accurate enthalpies than standard DFT when the $U_{\text {eff }}$ 's used were within the ranges of single-structure optimized $U_{\text {eff' }}$ 's (0.65- $0.9 \mathrm{eV}$ for $\mathrm{Np}$ and 1-1.5 eV for $\mathrm{U}$ ) - for example, the multi-structure optimized $U_{\text {eff' }}$ 's $(0.9 \mathrm{eV}$ for $\mathrm{Np}$ and $1.24 \mathrm{eV}$ for $\mathrm{U})$-determined in previous $\mathrm{U}-\mathrm{Zr}$ and $\mathrm{Np}-\mathrm{Zr}$ studies. Finally, we focused on the intermediate phase $\zeta(\mathrm{Np}, \mathrm{U})$, which at present is poorly characterized by experiments and unsatisfactorily described in the CALPHAD model. Based on ab initio calculated formation enthalpies for ordered end members and the compound energy formalism, we predicted its site occupations as function of compositions and temperature. Our study demonstrated that $U_{\text {eff }}$ 's fitted for $U$ and $\mathrm{Np}$ determined in the $\mathrm{U}-\mathrm{Zr}$ and $\mathrm{Np}-\mathrm{Zr}$ systems may be transferable to the $\mathrm{Np}-\mathrm{U}$ system and also provided new prediction of the site occupations for $\zeta(\mathrm{Np}, \mathrm{U})$, which may serve as reference for future experimental and modeling study of the $\mathrm{Np}-\mathrm{U}$ system.
\end{abstract}




\section{Introduction}

This work is motivated by the need to better understand the phase stability of the U$\mathrm{Pu}-\mathrm{Zr}-\mathrm{MA}(\mathrm{MA}=$ minor actinides $\mathrm{Np}, \mathrm{Am}$ and $\mathrm{Cm}$ ) metallic alloy systems for their safe, effective, and economical use as metallic nuclear fuels[1]. Previously we developed a thermodynamic model using CALculation of PHase Diagram (CALHAD) method and validated the $a b$ initio approaches of density functional theory (DFT) in its standard form[2,3] and one of its so-called DFT plus Hubbard $U(\mathrm{DFT}+U)$ modifications[4] for each of the $\mathrm{U}-\mathrm{Zr}[5,6]$ and the $\mathrm{Np}-\mathrm{Zr}[7]$ binary systems.

Here we continue to work on the $\mathrm{Np}-\mathrm{U}$ system, a binary whose both components are actinides. Unlike $\mathrm{U}-\mathrm{Zr}$ and $\mathrm{Np}-\mathrm{Zr}, \mathrm{Np}-\mathrm{U}$ has only been rarely studied despite the importance of this system being also a sub-system of the multicomponent U-Pu-Zr-MA metallic fuel system. Our literature survey identified only one systematic experimental phase diagram study[8] of $\mathrm{Np}-\mathrm{U}$, which was performed over 50 years ago. Moreover, to our knowledge only Kurata published a full thermodynamic assessment of this system using CALPHAD (a recent version is in Ref. [9]). We thus hope to further the understanding of this system through $a b$ initio calculations. However, $f$-electrons containing actinides are challenging to treat. For example, in our previous study of the UZr system[5] we found that based on generalized gradient approximation (GGA) standard DFT with and without spin orbit coupling (SOC) included on average overestimates the enthalpy of formation by about 0.07 and $0.10 \mathrm{eV} /$ atom ( 7 and $10 \mathrm{~kJ} / \mathrm{mole})$, respectively. In comparison, through fitting $U_{\text {eff }}$ to existing experimental and computational data, in particular enthalpies from reliably assessed CALPHAD models, we found DFT $+U$ could reduce the errors to about 0.02 and $0.04 \mathrm{eV} /$ atom (2 and $4 \mathrm{~kJ} / \mathrm{mole}$ ). Similar behavior is found in the Np-Zr system[7], where the corresponding DFT errors are about 0.15 and $0.17 \mathrm{eV} /$ atom (15 and $17 \mathrm{~kJ} / \mathrm{mole}$ ) with and without SOC respectively, and while DFT $+U$ could reduce them to 0.03 and $0.06 \mathrm{eV} /$ atom ( 3 and $6 \mathrm{~kJ} / \mathrm{mole}$ ). While these results seem encouraging, we face several open questions when dealing with this new Np-U system.

The first question is whether DFT $+U$ is applicable to $U$ and Np and their metallic alloy systems. Söderlind et al. recently criticized[10, 11] our U-Zr studies arguing that it is fundamental incorrect to model U-Zr and other related alloy systems using DFT $+U$ as they believed that DFT $+U$ predicts a) too large volume, b) too small bulk modulus, $\mathrm{c}$ ) energetics contradicting the phase diagram and d) unphysical magnetic moments. We demonstrated in our replies[12-14] that their arguments a) - c) were either incorrect or inadequate to challenge the applicability of DFT $+U$ because they focused on only $\gamma \mathrm{U}$ and/or $\gamma(\mathrm{U}, \mathrm{Zr})$ at $U_{\text {eff }}=1.24 \mathrm{and} / \mathrm{or} 2 \mathrm{eV}$ while neglected the majority of our evidence for all the other phases and at other $U_{\text {eff }}$ values (more on $U_{\text {eff }}$ in Section 2.2). However, we share the concern of Söderlind et al. that DFT $+U$ even at fitted $U_{\text {eff }}$ may predict incorrect magnetic state for selected cases, the resolution of which is an ongoing area of research. Nevertheless, we demonstrated[12] that DFT $+U$ could still provide meaningfully improved results of other properties for $\mathrm{U}-\mathrm{Zr}$, in particular energetics, even if not accounting the magnetic moments correctly. 
This study focuses only on energetics so if we accept that DFT $+U$ may be applicable in this case, the main question then becomes what $U_{\text {eff }}$ should we use in the Np-U system. Are the same "optimal" (See section 2.2) $U_{\text {eff }}$ values for $\mathrm{Np}$ and $\mathrm{U}$ that we established through energetic fitting in the $\mathrm{U}-\mathrm{Zr}[5,6]$ and $\mathrm{Np}-\mathrm{Zr}[7]$ systems transferable to the $\mathrm{Np}-\mathrm{U}$ system? The answer to this question is critical to determining if the DFT $+U$ approach with $U_{\text {eff }}$ established in a few benchmark systems can be applied more broadly on other similar metallic fuel systems through use of transferable $U_{\text {eff }}$ values.

In the next sections we will first assess existing experimental and theoretical results to identify phase fields that have been reliably characterized and modeled. We will then focus on the energetics underlying these robustly modeled phase fields - the four terminal and the body-centered cubic (BCC) solution phases to clarify the open question whether $U_{\text {eff }}$ is transferable. Finally, we consider the more uncertain phase field $\zeta(\mathrm{Np}, \mathrm{U})$ with the aim of gaining some new understanding through $a b$ initio predictions. The remainder of this manuscript proceeds as follows. Section 2 reviews the existing experimental and CALPHAD studies and the "optimal $U_{\text {eff }}$ " values from our previous U$\mathrm{Zr}$ and $\mathrm{Np}-\mathrm{Zr}$ studies. Section 3 describes the details of our methodology. Section 4 presents and discusses the results. Finally, Section 4 summarizes the conclusions.

\section{Literature Review}

\subsection{Existing phase stability studies}

The Np-U system contains 6 stable solid phases in its current ambient pressure phase diagram, as summarized in Table 1. As mentioned in the Introduction, as far as we are aware there is only one experimental phase diagram study[8] and one full CALPHAD assessment[9] of this binary system. Mardon and Pearce[8] investigated the Np-U equilibrium diagram and the obtained data are summarized in Figure 1. Among them thermal analysis and dilatometry data points should be on the phase boundary, while Xray data only indicate whether the points are in a single- or two-phase field, and may not necessarily be on phase boundary. As shown in Figure 1, Mardon and Pearce[8]'s data show that $\gamma \mathrm{Np}$ and $\gamma \mathrm{U}$ are completely miscible with each other, forming a single BCC solution phase $\gamma(\mathrm{Np}, \mathrm{U})$. The solidus and liquidus are quite close and both of them are almost straight lines connecting the melting point of $\mathrm{Np}$ and $\mathrm{U}$. Such features of phase diagram are consistent with the two elements forming very close to ideal solution in both the liquid and the BCC phases and each having quite similar and small entropies of melting. Also, the solubility of $U$ in $\beta(\mathrm{Np})$, and of $\mathrm{Np}$ in $\alpha(\mathrm{U})$ and $\beta(\mathrm{U})$ are quite large and the only intermediate phase $\zeta(\mathrm{Np}, \mathrm{U})$ is stable over large ranges of composition and temperature. Correspondingly, the two-phase fields between $\zeta$ and the four terminal solution phases are very small.

For the intermediate phase $\zeta(\mathrm{Np}, \mathrm{U})$, Mardon and Pearce[8] argued based on their Xray diffraction results that its crystal structure is simple cubic and it is isomorphous with $\zeta(\mathrm{Pu}, \mathrm{U})$ phase in the $\mathrm{Pu}-\mathrm{U}$ system. However, in a more recent study Lawson et al.[15] determined with neutron powder diffraction that $\zeta(\mathrm{Pu}, \mathrm{U})$ actually crystallizes in a much

more complex structure-in space group $\mathrm{R} \overline{3} \mathrm{~m}$ (no.166) with 58 atoms in the primitive 
unit cell occupying 10 distinct lattice sites in the asymmetric unit. Some structural information for the 10 sites is provided in Table 2 following Ref.[15]. Coordination number therein was defined as the number of neighboring atoms within $3.7 \AA$ from the central atom on a site and their values are quoted from Lawson et al.[15]. The choice of $3.7 \AA$ as cutoff was rationalized by the fact that distances to neighboring atoms outside the cutoff jump abruptly from smaller than 3.7 to larger than $4.4 \AA$, implying those atoms are not well-bonded with the central atoms. Wyckoff label and atomic environment (as defined in Ref.[16]) for each site were not assigned in the original article by Lawson et al. [15] but only later in Ref.[17], and here also provided in Table2. Despite significant knowledge of the $\zeta(\mathrm{Pu}, \mathrm{U})$ structure, the crystal structure of $\zeta(\mathrm{Np}, \mathrm{U})$ has, to our knowledge, never been systematically explored beyond the initial study of Mardon and Pearce[8].

Figure 1 shows the phase diagram of $\mathrm{Np}-\mathrm{U}$ predicted by the CALHAD model of Kurata[9], which was developed based on Mardon and Pearce's data. We find that Kurata's model reproduces the liquidus, solidus in addition to the BCC phase boundaries rather satisfactorily. However, it compares less favorably with the experimental data at low temperature, in particular near the Np-rich side. Some differences include: 1) on bottom left, CALPHAD results show a very large two-phase field of $\alpha(\mathrm{Np})+\zeta(\mathrm{Np}, \mathrm{U})$ that overlaps with most of the thermal analysis experimental points (black square) expected to correspond to the phase boundaries of $\alpha(\mathrm{Np})+\beta(\mathrm{Np})$ two-phase field. 2) On the middle left, the CALPHAD phase boundary for the two phase field of $\zeta(\mathrm{Np}, \mathrm{U})+\beta(\mathrm{Np})$ seems to be of opposite curvature on the left side from the transition line that may be expected between experimental single and two phase X-ray data. However, as X-ray data are not necessarily on the phase boundary, we must compare if the single- and two-phase data are located in the corresponding appropriate phase fields. We see that $\zeta(\mathrm{Np}, \mathrm{U})+\beta(\mathrm{Np})$ two-phase field from CALPHAD correctly covers all the two-phase data (cyan star) expected to be in $\zeta(\mathrm{Np}, \mathrm{U})+\beta(\mathrm{Np})$ but also includes many of the X-ray single-phase data points (magenta triangle). 3) Finally, on the right, the CALPHAD two-phase field of $\alpha(\mathrm{U})_{+} \zeta(\mathrm{Np}, \mathrm{U})$ also covers most of the single phase X-ray data. In short, Kurata's model seems to reproduce experimental phase boundary data well at high temperature. At low temperature, it also captures $\beta(\mathrm{U})$ phase boundaries well but shows some discrepancies for those of $\zeta(\mathrm{Np}, \mathrm{U}) \alpha(\mathrm{Np}), \beta(\mathrm{Np})$ and $\alpha(\mathrm{U})$. We believe the later discrepancies may be due to potentially very large uncertainty in the experimental data, which were obtained using "a very limited amount of neptunium"[8] that could particularly cause problem for phases stable at low temperature due to difficulty in reaching equilibrium. The discrepancies could also be caused by describing the multi-site intermediate compound phase $\zeta(\mathrm{Np}, \mathrm{U})$ with a simple solution model[9]. Either way, this analysis suggests that we could use the more reliably modeled $\mathrm{BCC}$ and terminal solutions phases to resolve the questions on transferability of $U_{\text {eff }}$ and then use $a b$ initio calculations to better understand the $\zeta(\mathrm{Np}, \mathrm{U})$ phase field, which is the approach we take below.

\section{2 "Optimal $U_{\text {eff" }}$}

In our previous discussions of DFT $+U$ results for the $\mathrm{U}-\mathrm{Zr}[5,6]$ and $\mathrm{Np}-\mathrm{Zr}$ binary

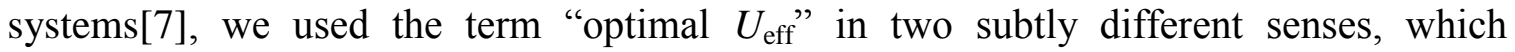
probably caused some confusion. Here we define explicit terminologies for each sense of 
"optimal $U_{\text {eff }}$ " and clarify their values. First, we define "single-structure optimized $U_{\text {eff }}$ "

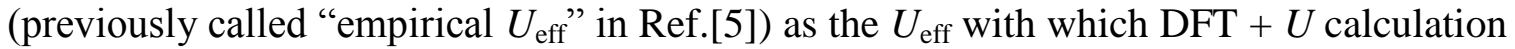
reproduces a specified physical observable (e.g., formation enthalpy) of a single material (i.e., crystal structure). In Refs.[5, 12], we found composition affects negligibly Hubbard $U$ of $U$ and $U-Z r$ that we estimated theoretically using the linear response approach[18] and different observable properties of a single material are roughly consistent, so approximately each single phase has a "single-structure optimized $U_{\text {eff }}$ ". As expected, single-structure optimized $U_{\text {eff }}$ varies for different phases, and for any system that contains more than one phase, single-structure optimized $U_{\text {eff }}$ 's for the individual phases are distributed within a range. Second, having to determine "single-structure optimized $U_{\text {eff }}$ " for each specific phase in the $\mathrm{U}$ and $\mathrm{U}-\mathrm{Zr}$ systems is laborious and not very practical for general modeling of these systems with DFT $+U$, so in Ref.[5] we fit $U_{\text {eff }}$ to give smallest average enthalpy error when considering multiple stable solid phases together. We name that single value of $U_{\text {eff }}$ "multi-structure optimized $U_{\text {eff }}$ " (previously called

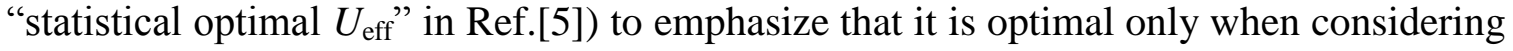
the average of multiple structures but may not be necessary so when only considering a single structure. We found the ranges of single-structure optimized $U_{\text {eff }}$ to be between about $0.99-1.49 \mathrm{eV}$ and $0.65-0.90 \mathrm{eV}$ for $\mathrm{U}$ and $\mathrm{Np}$ for the multiple solid phases in the U$\mathrm{Zr}[5]$ and $\mathrm{Np}-\mathrm{Zr}[7]$ systems, respectively. For the BCC phase, we found its singlestructure optimized $U_{\text {eff }}$ to be roughly $0.99 \mathrm{eV}$ and $0.65 \mathrm{eV}$ for $\mathrm{U}$ and $\mathrm{Np}$, in the $\mathrm{U}-\mathrm{Zr}[5$, 12] and $\mathrm{Np}-\mathrm{Zr}$ [7] systems, respectively. Note for the U-Zr system, we used $J=0.51$ so the somewhat awkward values of 0.99 and $1.49 \mathrm{eV}$ (from $U-J=1.50-0.51$ and 2.000.51 , respectively) are the $U_{\text {eff }}$ 's that were actually the values used, but often their rounded values of 1 and $1.5 \mathrm{eV}$ were used in our discussion. In terms of multi-structure optimized $U_{\text {eff }}$, we found the values to be about $1.24 \mathrm{eV}$ and $0.90 \mathrm{eV}$ for $\mathrm{U}$ and $\mathrm{Np}$ in the $\mathrm{U}-\mathrm{Zr}[5]$ and $\mathrm{Np}-\mathrm{Zr}$ [7] systems, respectively. Note that the BCC phase was not included when determining the multi-structure optimized $U_{\text {eff }}$ in order to avoid the significant noise that would be introduced due to both the uncertainty introduced by performing only constrained structural relaxation necessary to mitigate the mechanical instability and also the very large discrepancies in the enthalpies from the reference CALPHAD models for the BCC phase in both the U-Zr and Np-Zr systems.

\section{Methodology}

Stable solid phases of the Np-U system were modeled in the structure, composition and setting given in Table 1 . Six solid phases are currently known to be stable under ambient pressure in the Np-U system: $\alpha(\mathrm{Np}), \beta(\mathrm{Np}), \alpha(\mathrm{U})$, and $\beta(\mathrm{U})$ terminal solution phase, BCC single solution phase, and intermediate phase $\zeta(\mathrm{Np}, \mathrm{U})$. The BCC phase is labeled $\gamma(\mathrm{Np}, \mathrm{U})$ here because it is the solution of $\gamma \mathrm{Np}$ and $\gamma \mathrm{U}$. Mardon and Pearce used $\delta$ to label the intermediate phase[8]. However, to distinguish that it has a different structure from the $\delta$ phases in the $\mathrm{U}-\mathrm{Zr}$ and $\mathrm{Np}-\mathrm{Zr}$ systems and recognizing that it was suggested to be isomorphous with $\zeta$ phase of $\mathrm{Pu}-\mathrm{U}[8]$, we labeled it $\zeta$ here. All these phases except $\zeta$ were modeled with supercells that were generated using the Special Quasi-random Structure (SQS) method [19] as implemented in the Alloy Theory Automated Toolkit (ATAT) [20]. These SQS supercells are the same as were used in our previous studies of the $\mathrm{Np}-\mathrm{Zr}$ and $\mathrm{U}-\mathrm{Zr}$ systems[5, 7]. The elemental reference structures $\alpha \mathrm{Np}, \beta \mathrm{Np}, \gamma \mathrm{Np}, \alpha \mathrm{U}$, 
$\beta \mathrm{U}$, and $\gamma \mathrm{U}$ had been calculated in $\operatorname{Refs}[5,7]$ and the results of the previous studies were used here whenever necessary.

All ab initio calculations were performed in the general framework of DFT[2, 3] using the Vienna $A b$ initio Simulation Package (VASP)[21, 22]. The electron-ion interaction was described with the projector-augmented-wave (PAW) method[23] as implemented by Kresse and Joubert[24]. The PAW potentials used treat $6 s^{2} 6 \mathrm{p}^{6} 7 \mathrm{~s}^{2} 5 \mathrm{f}^{4} 6 \mathrm{~d}^{1}$ and $6 s^{2} 6 p^{6} 7 s^{2} 5 f^{3} 6 d^{1}$ as valence electrons for $\mathrm{Np}$ and $\mathrm{U}$, respectively. The exchangecorrelation functional parameterized in the generalized gradient approximation (GGA)[25] by Perdew, Burke and Ernzerhof (PBE)[26] was used. The stopping criteria for self-consistent loops used were $0.1 \mathrm{meV}$ and $1 \mathrm{meV}$ tolerance of total free energy for the electronic and ionic relaxation, respectively. Cutoff energy of $450 \mathrm{eV}$ was used throughout all calculations. The partial occupancies were set using the Methfessel-Paxton method[27] of order one with a smearing width of $0.2 \mathrm{eV}$. The electronic and ionic optimizations were performed using a Davidson-block algorithm[28] and a Conjugategradient algorithm[29], respectively.

All systems except $\gamma(\mathrm{Np}, \mathrm{U})$ were fully optimized in terms of volume, lattice shape and ion positions. However, such relaxation could not be done for $\gamma(\mathrm{Np}, \mathrm{U})$ as it is mechanically unstable[5]. Following our previous practice to mitigate the mechanical instability of $\mathrm{BCC} \mathrm{U}-\mathrm{Zr}$ and $\mathrm{Np}-\mathrm{Zr}[5,7]$, only volume relaxation was performed for $\mathrm{BCC}$ $\gamma(\mathrm{Np}, \mathrm{U})$ structures, with no cell-internal relaxations. The $\mathrm{BCC}$ volume mismatch between $\mathrm{Np}$ and $\mathrm{U}$ are about $13 \%$ from standard DFT. DFT $+U$ predicts larger volumes for Np and $\mathrm{U}$, and the volume mismatch reduces to $6 \%$ for example at $\left(U_{\text {eff }}(\mathrm{Np}), U_{\text {eff }}(\mathrm{U})\right)=(0.6$, $0.99) \mathrm{eV}$, which are the single-structure optimized $U_{\text {eff }}$ 's for BCC Np and U. To assess the impact of ignoring cell-internal relaxations on the energy we fit the energy of cellinternal relaxations as a function of volume mismatch for the 10 BCC binary alloys formed by V, Nb, Ta, Mo and W (See Supplementary Materials Table S1 and Figure S1), all of which are stable in the BCC structure and can therefore be fully relaxed (unlike U$\mathrm{Np}$ ). From this fit we found that the overestimation in enthalpies of mixing due to the lack of ion relaxation corresponding to the volume mismatches of standard DFT and DFT $+U\left(\right.$ at $\left.\left(U_{\text {eff }}(\mathrm{Np}), U_{\text {eff }}(\mathrm{U})\right)=(0.6,0.99) \mathrm{eV}\right)$ were expected to be approximately 0.013 and $0.006 \mathrm{eV} / \mathrm{atom}$, respectively at 50 at.\%. At other compositions, the corresponding energies should be smaller or at most comparable, as evidenced by the testing results at 25at.\% (0.008 and $0.005 \mathrm{eV} /$ atom) and 75 at.\% (0.012 and $0.006 \mathrm{eV} /$ atom). These results showed that the effect of ion relaxation should be quite small comparable to the intrinsic uncertainty of the $a b$ initio and CALPHAD modeling approaches. However, we will see that the enthalpy of mixing for BCC Np-U is also small. We will discuss the physical implication of neglecting ion relaxation in the next section.

Spin polarization was included in all calculations although spin-orbit coupling (SOC) was not. Magnetic moments for all calculations are given in Supplementary Materials Figures S2-S4. In our previous validations on $\mathrm{U}-\mathrm{Zr}$ and Np-Zr binaries, SOC was found to affect the calculated enthalpy by about 0.02 and $0.03 \mathrm{eV} /$ atom [5, 7], respectively, which were relatively small compared to the differences in the average errors in enthalpy between standard DFT and DFT $+U$-about 0.06 and $0.10 \mathrm{eV} /$ atom for $\mathrm{U}-\mathrm{Zr}$ and Np-Zr at the multi-structure optimized $U_{\text {eff }}$ values. While we understand that small changes due 
to SOC can still have a qualitative effect for selected cases, e.g., changing the sign of DFT $+U$ enthalpy of mixing for $\gamma(\mathrm{U}, \mathrm{Zr})$ at $U_{\mathrm{eff}}(\mathrm{U})=1.24 \mathrm{eV}[5]$ (as the enthalpy is almost 0 ), our objective here was mainly to assess the relative accuracy of standard DFT and DFT $+U$. We thus did not include SOC here to reduce the computational costs. Future work can be performed to include SOC and decide what is the best absolute accuracy that DFT $+U$ can attain.

The DFT $+U$ functional proposed by Dudarev et al.[4] was used. This form of DFT $+U$ does not introduce explicit local exchange $J$ term but only an effective Hubbard $U$ term that depends on $U_{\text {eff }}=U-J$. This approach also recovers the standard DFT functional exactly when $U_{\text {eff }}=0$. DFT $+U$ potential is applied both on $U$ and $\mathrm{Np}$ sites. For historical reasons we did not set set $J=0$ as one conventionally does but instead to 0.6 and $0.51 \mathrm{eV}$ for $\mathrm{Np}$ and $\mathrm{U}$, respectively and vary $U$ up to $3 \mathrm{eV}$. This detail is provided here merely to explain why we discussed results at some awkward $U_{\text {eff }}$ values like 0.99 and 1.24 , as these are $U_{\text {eff }}=1.5-0.51$ and $U_{\text {eff }}=1.75-0.51$. One can reproduce our results as long as the same $U_{\text {eff }}$ 's are used, regardless of what specific pair of $U$ and $J$ is used to reach the

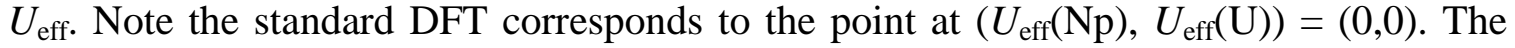
metastable solution issue of $\mathrm{DFT}+U$ is combated using the $U$-ramping method[30] with modifications described in Ref.[5].

Neglecting the pressure dependence, enthalpy of formation for a given alloy was defined as:

$$
E^{\text {form }}\left(\mathrm{Np}_{x_{\mathrm{Np}}} \mathrm{U}_{x_{\mathrm{U}}}\right)=E\left(\mathrm{~Np}_{x_{\mathrm{Np}}} \mathrm{U}_{x_{\mathrm{U}}}\right) \quad x_{\mathrm{Np}} E(\mathrm{~Np}) x_{\mathrm{U}} E(\mathrm{U})
$$

where $E$ is total energy per unit amount (e.g., per atom), $\mathrm{Np}_{x_{\mathrm{Np}}} \mathrm{U}_{x_{\mathrm{U}}}$ and $\alpha \mathrm{Np}(\alpha \mathrm{U})$ are the alloy and the constituent elemental metal references for $\mathrm{Np}(\mathrm{U})$, respectively, and $x_{\mathrm{Np}}$ $\left(x_{\mathrm{U}}\right)$ are the mole fractions of $\mathrm{Np}(\mathrm{U})$ in the alloy with $x_{\mathrm{Np}}+x_{\mathrm{U}}=1$.

Eq. (1) should be slightly modified for enthalpy of mixing $E^{\mathrm{mix}}$, which was defined as in Eq. (1) except by referencing to elemental $\mathrm{Np}$ and $\mathrm{U}$ in the structure of the alloy phase in place of $\alpha \mathrm{Np}$ and $\alpha \mathrm{U}$. For example, for BCC $\gamma(\mathrm{Np}, \mathrm{U})$ the references should be BCC $\gamma \mathrm{Np}$ and $\gamma \mathrm{U}$.

The dilute heat of solution was defined as:

$$
E^{\mathrm{dil}}=\lim _{x \rightarrow 0} \frac{E^{\mathrm{form}}}{x} \approx \frac{E^{\mathrm{form}}}{x}(\text { when } x \square 1)
$$

where enthalpy of formation was defined in Eq. (1), and $x$ is the concentration of the dilute solute specie. The limit could be approximately removed at small $x$ and in our case we used $x_{\text {solute }}=1 / 16$ for $\alpha(\mathrm{Np}), \beta(\mathrm{Np}), \alpha(\mathrm{U})$, and $\beta(\mathrm{U})$.

In this work we assumed that $\zeta(\mathrm{Np}, \mathrm{U})$ is indeed isomorphous with $\zeta(\mathrm{Pu}, \mathrm{U})$ and used the presently accepted 58-atom rhombohedral primitive unit cell structure of $\zeta(\mathrm{Pu}, \mathrm{U})$ 
according to Lawson et al.[15]. Even with this assumption, we were still left with unknown site occupations for all compositions in the stable range of $\zeta(\mathrm{Np}, \mathrm{U})$. We explored site occupations with the following approach. First, we noted that enumerating all possible combinations of occupations of the 10 lattice sites was not tractable at present - even only considering cases with full occupation of either $\mathrm{Np}$ or $\mathrm{U}$ on each site requires calculations of $2^{10}=1024$ structures. Therefore, we first grouped the 10 lattice sites into 5 groups based on similar coordination number/atomic environment. As listed in Table 3: group I includes sites 1, 3 and 7, group II includes site 2, 5, 6, group III is site 4 , group IV is site 8 , and group V includes sites 9, 10. Coordination number has been shown to be an effective criterion for grouping lattice site of $\sigma$ phase in many transition metal alloy systems, for example as demonstrated in the comparison of sublattice models due to different site grouping in the Mo-Re system[31]. Furthermore, atomic environment considers symmetry in addition to the total number of the nearest neighbors of a lattice site, so two or more lattice sites may have different atomic environment even when they have the same coordination number[32]. As a result, atomic environment may be an even more effective site grouping criterion than just coordination number. In the present case of $\zeta$ each site has a unique set of both coordination number and atomic environment and hence the same grouping is arrived based on either coordination number or atomic environment. Alternative ways of grouping are possible, but given the uncertainty in $a b$ initio methods and also considering the limited experimental data available for validation at this point, we did not pursue them in this study.

After the grouping, we assumed that chemical ordering of $\zeta(\mathrm{Np}, \mathrm{U})$ is fully represented by the $2^{5}=32$ end members where each group of sites are either only occupied with $\mathrm{U}$ or with $\mathrm{Np}$. Gibbs free energy of formation $G^{\text {form }}$ for $\zeta(\mathrm{Np}, \mathrm{U})$ alloy is then a function of occupations $\left\{y_{\mathrm{Np}}^{g}\right\}$, the optimal values of which are functions of composition $x_{\mathrm{Np}}$ and temperature $T$. We modeled the Gibbs free energy parametrized by site occupation and temperature with the compound energy formalism (CEF) [33, 34]:

$$
G^{\text {form }}\left[\left\{y_{\mathrm{Np}}^{g}\left(x_{\mathrm{Np}}, T\right)\right\}\right]={ }^{\text {ref }} G^{\text {form }}+{ }^{\text {id }} G^{\text {mix }}+{ }^{\text {ex }} G^{\text {mix }}
$$

where $g \quad\{\mathrm{I}, \mathrm{II}, \mathrm{III}, \mathrm{IV}, \mathrm{V}\}$ is group label, ${ }^{\mathrm{ref}} G^{\text {form }}\left(x_{\mathrm{Np}}, 0\right)=\sum_{i, j, k, l, m \in \mathrm{Np}, \mathrm{U}\}} y_{i}^{\mathrm{I}} y_{j}^{\mathrm{II}} y_{k}^{\mathrm{III}} y_{l}^{\mathrm{IV}} y_{m}^{\mathrm{V}} E_{i j k l m}^{\text {form } 0}$ is the so-called Gibbs free energy surface of reference, which is the average enthalpy of formation that is calculated as sum of the enthalpy of formation $E_{i j k m}^{\mathrm{form}, 0}$ of end member $i j k l m$ weighted by products of the occupations $y_{i}^{\mathrm{I}} y_{j}^{\mathrm{II}} y_{k}^{\mathrm{III}} y_{l}^{\mathrm{IV}} y_{m}^{\mathrm{V}}$ for all the 32 ordered end members, ${ }^{i d} G^{\mathrm{mix}}\left(x_{\mathrm{Np}}, T\right)=T^{i d} S^{\mathrm{mix}}\left(x_{\mathrm{Np}}, T\right)$ is the ideal configurational mixing free energy with ${ }^{\text {id }} S^{\text {mix }}=R \sum_{g} a^{g} \sum_{i \in \mathrm{Np}, \mathrm{U}\}} y_{i}^{g} \ln \left(y_{i}^{g}\right)$ and $a^{g}$ being the fraction of all lattice sites in group $g$ (see Table 3), and finally ${ }^{\text {ex }} G^{\text {mix }}$ is excess Gibbs free energy, primarily due to interactions between atoms occupying sites in the same group. In this study we assumed ${ }^{\text {ref }} G^{\text {mix }}\left(x_{\mathrm{Np}}, 0\right)={ }^{\text {ref }} G^{\text {mix }}\left(x_{\mathrm{Np}}, T\right)$ and ${ }^{\mathrm{ex}} G^{\text {mix }}=0$. The second assumption could be justified by the fact that the fraction of near neighbor bonds between atoms from the same group $f_{\mathrm{g}}$ 
(given in Table 3$)$ is small for most groups. At given $\left(x_{\mathrm{Np}}, T\right)$, minimization of $G^{\text {form }}$ with respect to $\left\{y_{\mathrm{Np}}^{g}\right\}$ subject to the constraint that $a^{g} y_{\mathrm{Np}}^{g}=x_{\mathrm{Np}}$ was performed in this study with MATLAB to obtain the optimal (i.e., equilibrium) occupations $\left\{\bar{y}_{\mathrm{Np}}^{g}\right\}$. We found that direct optimization of objective function $G^{\text {form }}$ as given in (3) was numerically difficult to converge and actually optimized instead $G^{\text {mix }}=G^{\text {form }} \sum_{i \in \mathrm{Np}, \mathrm{U}\}} x_{i} E_{\text {iiiiii }}^{\mathrm{form}, 0}$, which shares the same optimization solution $\left\{\bar{y}_{\mathrm{Np}}^{g}\right\}$ as $G^{\text {form }}$.

\section{Results and Discussion}

\subsection{Are $U_{\text {eff's }}$ Transferable?}

As we mentioned in the Introduction, a major question we hope to address is whether $\mathrm{DFT}+U$ with the $U_{\text {eff }}$ 's for $\mathrm{Np}$ and $\mathrm{U}$ fitted individually in the $\mathrm{Np}-\mathrm{Zr}$ and the $\mathrm{U}-\mathrm{Zr}$ systems (reviewed in section 2.2) are transferable to the $\mathrm{Np}-\mathrm{U}$ system. For this purpose, we first focus on the terminal solution and the BCC phases and use enthalpies from the CALPHAD model as references to validate different $U_{\text {eff }}$ 's in this section.

Before we start, it is essential to note first that both CALPHAD and ab initio enthalpies have their own intrinsic error bars. On the CALPHAD side, from the comparison above, the CALPHAD model shows some discrepancy from the experimental data at low temperature for $\zeta(\mathrm{Np}, \mathrm{U}), \alpha(\mathrm{Np}), \beta(\mathrm{Np})$ and $\alpha(\mathrm{U})$. At high temperature, even though the model reproduces the experimental phase boundaries for the BCC phase rather satisfactorily, some degrees of freedom are still allowed in how the total free energy is partitioned between enthalpy and entropy and how large the total free energy is, which depend on the state of the liquid phase that is in equilibrium with the $\mathrm{BCC}$ phase. For example, in our recent work on $\mathrm{U}-\mathrm{Zr}$ we found that two earlier CALPHAD models[35, 36] predicted similar BCC phase boundaries as our model[6], however the enthalpy of mixing for the BCC phase from them were about 0.08-0.09 eV/atom (7.7-8.8 $\mathrm{kJ} /$ mole) larger at 50. at $\% \mathrm{Zr}$ than that from our model. One of the models[35] also predicted the excess entropy of mixing for the liquid phase to be about $0.10 \mathrm{eV} / \mathrm{atom} / \mathrm{K}(10 \mathrm{~kJ} / \mathrm{mole} / \mathrm{K})$ larger than the other two[6, 36]. On the ab initio side, standard DFT was found, on average, to overestimate the CALPHAD predicted enthalpies by about 0.10 and $0.15 \mathrm{eV} /$ atom $(9.7$ and $15.4 \mathrm{~kJ} / \mathrm{mole})$ for $\mathrm{U}$ metal and $\mathrm{U}-\mathrm{Zr}$ alloy[5] and Np metal Np-Zr alloy[7], respectively. DFT $+U$ was found to reduce the difference, but not to zero. The smallest average difference between DFT $+U$ and CALPHAD enthalpies for the U-Zr system[5] was found at $U_{\text {eff }}(\mathrm{U})=1.24 \mathrm{eV}$ (i.e., multistructure optimized $U_{\text {eff }}$, which was still about 0.02-0.03 eV/atom $(1.9-2.9 \mathrm{~kJ} / \mathrm{mole})$ depending on which CALPHAD model we compared to; for the $\mathrm{Np}-\mathrm{Zr}$ system, the corresponding smallest average difference was found to be 0.03-0.06 eV/atom (2.9-5.8 $\mathrm{kJ} / \mathrm{mole}$ ) at the multi-structure optimized $U_{\text {eff }}(\mathrm{Np})=0.90 \mathrm{eV}$. Given these uncertainties we can expect similar error bars in the present comparisons. In consequence, we need to 
make comparisons across as many measured values as possible to avoid being misled by errors in either the $a b$ initio or the CALPHAD part.

We start with the four terminal solution phases $\alpha(\mathrm{Np}), \beta(\mathrm{Np}), \alpha(\mathrm{U})$, and $\beta(\mathrm{U})$, as shown in Figure 2. We first discuss the qualitative trend of how their dilute heats of solution are dependent on $U_{\text {eff }}(\mathrm{Np})$ and $U_{\text {eff }}(\mathrm{U})$. A first observation is that the dilute heat of solution is negligibly affected by $U_{\text {eff }}(\mathrm{U})$ for the two Np-rich systems in panels a) and b) and by $U_{\text {eff }}(\mathrm{Np})$ for the two U-rich systems in panels c) and d), respectively. More specifically, in panels a) and b), both the systems contain 6.3 at. $\% \mathrm{U}$ and their dilute heats of solution calculated at the same $U_{\text {eff }}(\mathrm{Np})$ are almost the same when $U_{\text {eff }}(\mathrm{U})$ varies in the range of 0-2.0 eV, and are only slightly larger at $U_{\text {eff }}(\mathrm{U})=2.5 \mathrm{eV}$. Similarly, panels c) and d) show that the two U-rich systems' dilute heats of solution change little at the same $U_{\text {eff }}(\mathrm{U})$ when $U_{\text {eff }}(\mathrm{Np})$ varies in the range of $0-1.40 \mathrm{eV}$. This general trend of dilute impurity $U_{\text {eff }}$ values having weak influence is presumably due to energy changes in the impurity atom with different $U_{\text {eff }}$ being largely canceled between the reactants and products in the substitution reaction. Note in c), an exception exists at $U_{\text {eff }}(\mathrm{U})=2 \mathrm{eV}$ with points for $U_{\text {eff }}(\mathrm{Np})=0.65$ and $0.90 \mathrm{eV}$ jumping somewhat discontinuously above the CALPHAD enthalpy planes, while all the other points are below. Considering that the general trend of dilute impurity $U_{\text {eff }}$ values having weak influence is so well followed in all other cases, we believe the two points above the CALPHAD planes in Fig. 2 c) are metastable solutions of DFT $+U$. Because $U_{\text {eff }}(\mathrm{U})$ for those points have exceeded the ranges of single-structure optimized $U_{\text {eff }}$ 's, they do not affect our validations and hence no further attempts beyond the approaches described in Sec. 2 have been made to find better solutions.

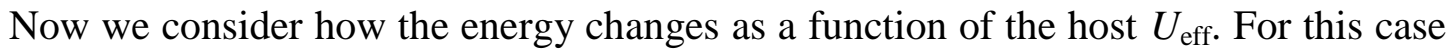
we observe three different behaviors. In Fig. 2 a) and b), the ab initio energy surfaces are almost horizontal planes when $U_{\text {eff }}(\mathrm{Np})$ is between 0 and $0.90 \mathrm{eV}$ and rise very steeply when it is larger. In c), the ab initio surface also essentially levels out between $U_{\text {eff }}(\mathrm{U})=0$ and $1.5 \mathrm{eV}$, drops between 1.5 and $2 \mathrm{eV}$, and rises afterwards. However, in d), the $a b$ initio surface drops continuously between $U_{\text {eff }}(\mathrm{U})=0$ and $2 \mathrm{eV}$ and rises afterwards. However, the gradient is different between the range of $0-1.5 \mathrm{eV}$ and the range of 1.5-2 $\mathrm{eV}$, again suggesting that above $U_{\mathrm{eff}}(\mathrm{U})=1.5 \mathrm{eV}$ the material behavior is quite different. At this point the physical origins of the three different behaviors are not clear.

Next we compare $a b$ initio dilute heats of solution with CALPHAD[9]. In panels a) c) of Figure 2, the ab initio values are essentially the same when $U_{\text {eff }}(\mathrm{Np})$ is between 0 $0.90 \mathrm{eV}$ and $U_{\text {eff }}(\mathrm{U})$ is between $0-1.5 \mathrm{eV}$-including the point of standard DFT at $U_{\text {eff }}(\mathrm{Np})=U_{\text {eff }}(\mathrm{U})=0$, all of which are very close to CALPHAD values. However, in panels d) for $\beta(\mathrm{U})$ at 96.7 at.\% $\mathrm{U}$, the dilute heat of solution from standard DFT (3.707 $\mathrm{eV} /$ solute atom) is too large compared to the CALPHAD value of $0.762 \mathrm{eV} /$ solute atom. $\mathrm{DFT}+U$ is found to predict lower enthalpy and improves the agreement with the CALPHAD. For example, if we take $U_{\text {eff }}(\mathrm{U})$ and $U_{\text {eff }}(\mathrm{Np})$ to be the multi-structure optimized $U_{\text {eff }}$ (i.e., $U_{\text {eff }}(\mathrm{U})=1.24 \mathrm{eV}$ and $U_{\text {eff }}(\mathrm{Np})=0.90 \mathrm{eV}$ ) we get a value of 1.350 $\mathrm{eV} / \mathrm{solute}$ atom, which is in much better good agreement with the CALPHAD value than DFT. 
The previous discussions on the terminal solution phases only look at one composition point for each phase. Next we consider BCC $\gamma(\mathrm{Np}, \mathrm{U})$ phase's enthalpy of mixing in Figure 3 over the whole composition range of 0 to 100 at.\% U. We show results calculated at $U_{\text {eff }}(\mathrm{U})=0,0.49,0.99$ and $1.49 \mathrm{eV}$ as black, red, green, and blue curves (with square, circle, upward triangle, and downward triangle symbols), respectively in each of the five panels of a) - e), which correspond to calculations at $U_{\text {eff }}(\mathrm{Np})=0,0.4,0.65,0.90$ and $1.40 \mathrm{eV}$, respectively. Again we start our discussion with qualitative features. First, the mixing enthalpy initially decreases and then increases when $U_{\text {eff }}(\mathrm{Np})$ changes from 0 to $1.40 \mathrm{eV}$. Second, when increasing $U_{\text {eff }}(\mathrm{U})$, the enthalpy increases in panels a) and b), essentially does not change in panel c), and decreases in panels d) and e). Third, both $U_{\text {eff }}(\mathrm{U})$ and $U_{\text {eff }}(\mathrm{Np})$ play a significant role in the enthalpy changes, but $U_{\text {eff }}(\mathrm{Np})$ has a somewhat larger effect than $U_{\text {eff }}(\mathrm{U})$. For example, the maximum change in enthalpy with $U_{\text {eff }}(\mathrm{Np})$ at fixed $U_{\text {eff }}(\mathrm{U})$ is about $0.15 \mathrm{eV} /$ atom across panels, while the maximum change in $U_{\text {eff }}(\mathrm{U})$ at fixed $U_{\text {eff }}(\mathrm{Np})$ is about $0.1 \mathrm{eV} /$ atom in panel e), and generally much less in all the other four panels of a) to d).

Now we compare $a b$ initio enthalpies to those predicted by Kurata's CALPHAD model[9], which are almost zero over the whole composition range. Figure 3 shows that standard DFT predicts the enthalpy of mixing for $\gamma(\mathrm{Np}, \mathrm{U})$ to be positive, about 0.020 eV/atom at the maximum near 25 at. $\% \mathrm{U}$, and this value is reasonably close to the CALPHAD values. In the Methodology, we estimated that due to the neglect of ion relaxation the calculated enthalpy of mixing for standard DFT should be reduced by about $0.013 \mathrm{eV} /$ atom at 50 at.\%. If we subtract the ion relaxation energy from standard DFT enthalpy of mixing, the resulting enthalpy will be smaller but should remain positive around 25 at.\%U. Positive enthalpies suggest possible existence of a miscibility gap, although the values are small enough that it may not be observable at the high temperatures where $\gamma(\mathrm{Np}, \mathrm{U})$ is stable. For DFT $+U$ enthalpies, we can see that those in panel e) are probably too large, in agreement with the results that $U_{\text {eff }}(\mathrm{Np})=1.40 \mathrm{eV}$ is larger than the range of single-structure optimized $U_{\text {eff }}(\mathrm{Np})$ range of $0.65-0.90 \mathrm{eV}$ from $\mathrm{Np}-\mathrm{Zr}$ comparisons. The single blue curve of $U_{\text {eff }}(\mathrm{U})=1.49 \mathrm{eV}$ in panel a) shows a maximum also near 25 at. $\% \mathrm{U}$ to be around $0.05 \mathrm{eV} /$ atom, which is probably also too large, in agreement with what we showed in Ref.[12] that although 1-1.5 eV is the general range of single-structure optimized $U_{\text {eff }}$ for overall modeling of $\mathrm{U}$ metal and $\mathrm{U}-\mathrm{Zr}$ alloys, the single-structure optimized $U_{\text {eff }}$ for BCC $\gamma \mathrm{U}$ and $\gamma(\mathrm{U}, \mathrm{Zr})$ phases is approximately $1 \mathrm{eV}$ (i.e., on the lower end of the range). If we take either the multistructure optimized $U_{\text {eff }}$ values determined over multiple stable solid phases of $\mathrm{U} / \mathrm{U}-\mathrm{Zr}$ and $\mathrm{Np} / \mathrm{Np}-\mathrm{Zr}$ systems (i.e., $U_{\text {eff }}(\mathrm{Np})=0.90 \mathrm{eV}$ [7] and $U_{\text {eff }}(\mathrm{U})=1.24 \mathrm{eV}[5]$ ), or the individual single-structure optimized $U_{\text {eff }}$ values for the BCC high temperature phases of $\gamma(\mathrm{U}, \mathrm{Zr})$ and $\gamma(\mathrm{Np}, \mathrm{Zr})$ (i.e., $U_{\text {eff }}(\mathrm{Np})$ near $0.65 \mathrm{eV}[7]$ and $U_{\text {eff }}(\mathrm{U})$ near $\left.0.99 \mathrm{eV}[12]\right)$, then our DFT $+U$ results suggest that the enthalpy of mixing for BCC $\gamma(\mathrm{Np}, \mathrm{U})$ is slightly negative. Further, the ion relaxation energy, although very small due to the reduced volume mismatch - estimated to be about $0.006 \mathrm{eV} / \mathrm{atom}$ at 50 at.\% U using the volumes calculated at $\left(U_{\text {eff }}(\mathrm{Np}), U_{\text {eff }}(\mathrm{Np})\right)=(0.6,0.99) \mathrm{eV}$, will also bring down the enthalpy slightly. Quantitatively, most of the DFT $+U$ curves in panels b) $-\mathrm{d}$ ) are also only about $0.02 \mathrm{eV} /$ atom or less away from the CALPHAD curves, which we expect to be within the uncertainty of these modeling approaches, as we discussed in the beginning of this section. Moreover, the negative enthalpy of mixing from DFT $+U$ in the whole 
composition range suggests that $\gamma(\mathrm{Np}, \mathrm{U})$ should have no miscibility gap, which is consistent with the fact that Mardon and Pearce did not find any evidence for the existence of a miscibility gap in their experiment[8], nor did the CALPHAD model[9] predicts one. In short, CALPHAD, standard DFT and DFT $+U$ predict almost zero, slightly positive and mostly slightly negative enthalpy of mixing for BCC $\gamma(\mathrm{Np}, \mathrm{U})$, respectively. Although they differ qualitatively by sign, the absolute differences are small in the sense that they are comparable with the error bars of these modeling approaches (within about $0.05 \mathrm{eV} /$ atom). We thus cannot tell which prediction is closer to the true situation except that the enthalpy of mixing for BCC $\gamma(\mathrm{Np}, \mathrm{U})$ is likely to be small, be it positive or negative. We hope future experimental studies can provide more constraints on these energies.

\subsection{Ab initio predictions for $\zeta(\mathrm{Np}, \mathrm{U})$}

After determining that DFT $+U$ using the same fitted $U_{\text {eff }}$ 's in the $\mathrm{U}-\mathrm{Zr}$ and $\mathrm{Np}-\mathrm{Zr}$ systems could provide improved or at least similar enthalpies in the Np-U system for phases that are believed to be reasonably modeled in the reference CALPHAD model, we now turn our attention to the remaining stable solid phase $\zeta(\mathrm{Np}, \mathrm{U})$ that is deemed to be poorly understood at present (see section 2.1).

Table 4 tabulates enthalpies of formation calculated for the 32 ordered end members of $\zeta(\mathrm{Np}, \mathrm{U})$ after the lattice site grouping detailed in Table 3, as well as the values predicted by $\mathrm{CEF}[33,34]$ at the 20 compositions where ordered end members exist, which are calculated based on the order end member enthalpies and optimized site occupations. The same results are also plotted in Figure 4. While for this phase CALPHAD enthalpies should be less reliable compared to the other phases above, at this moment we still provide the values at $300 \mathrm{~K}$ from the CALPHAD model[9] as reference. Note we used $\left(U_{\text {eff }}(\mathrm{Np}), U_{\mathrm{eff}}(\mathrm{U})\right)=(0.65,1.24) \mathrm{eV}$ in the DFT $+U$ calculations. Namely, for $\mathrm{U}$ we used the multi-structure optimized $U_{\text {eff }}$ but for $\mathrm{Np}$ we used $0.65 \mathrm{eV}$, which is the lower end for the range of sing-structure optimized $U_{\text {eff. }}$ The reason we did not use the multi-structure optimized $U_{\text {eff }} 0.90 \mathrm{eV}$ was that in the $\mathrm{Np}-\mathrm{Zr}$ system[7] $\mathrm{DFT}+U$ at $U_{\text {eff }}(\mathrm{Np})=0.90 \mathrm{eV}$ predicts wrong curvature for the enthalpy-composition curve of the corresponding intermediate compound phase $\delta(\mathrm{Np}, \mathrm{Zr})$, and it is possible that this issue may also exists for $\zeta(\mathrm{Np}, \mathrm{U})$. Believing also that the differences between $U_{\text {eff }}(\mathrm{Np})=0.65$ and 0.9 on formation enthalpy and site occupations should be small, we did not attempt to perform calculations at $\left(\left(U_{\text {eff }}(\mathrm{Np}), U_{\text {eff }}(\mathrm{U})\right)=(0.90,1.24) \mathrm{eV}\right)$.

Table 4 and Figure 4 show the following results. First, DFT, DFT $+U$ and CALPHAD all predict that enthalpy of formation for $\zeta(\mathrm{Np}, \mathrm{U})$ generally increases with mole fraction of $\mathrm{Np}$ (although not monotonically for the CALPHAD data), which suggests that $\zeta$ is probably easier to form on the U-rich side. Second, the DFT CEF enthalpy curve lies significant higher above the CALPHAD curve while DFT $+U$ curve is lower and much closer. As an example, Table 4 lists that DFT predicts the enthalpy of formation to span between 0.091 (at $x(\mathrm{~Np})=0$ ) and 0.276 (at $x(\mathrm{~Np})=1$ ) eV/atom. Such large positive formation enthalpy values are probably too high for a compound phase that is stable at room temperature. The situation is the same for the room temperature stable compound phase $\delta$ in the $\mathrm{U}-\mathrm{Zr}$ and Np-Zr systems, for which we found DFT also predicts 
large positive formation enthalpies. As a reference, CALPHAD values are 0.004 and $0.052 \mathrm{eV} /$ atom at $x(\mathrm{~Np})=0$ and 1 , respectively and the minimum is about -0.003 $\mathrm{eV} /$ atom around $x(\mathrm{~Np})=0.5$. In comparison, DFT $+U$ predicts much smaller formation enthalpy spanning between $0.038($ at $x(\mathrm{~Np})=0$ ) and 0.136 (at $x(\mathrm{~Np})=1$ ) eV/atom. Another aspect to compare is the difference in enthalpy between the two ends at $x(\mathrm{~Np})=$ 0 and 1. DFT, DFT $+U$ and CALPAHD predict values of $0.185,0.098$ and 0.048 eV/atom, respectively. On average, the root mean square (RMS) of enthalpy differences between DFT CEF and CALPHAD at the 20 compositions where ordered end members exist is $0.155 \mathrm{eV} /$ atom, while that between DFT $+U$ CEF and CALPHAD is 0.055 eV/atom. All these comparisons show that DFT $+U$ should improve the accuracy of enthalpy compared to DFT for $\zeta(\mathrm{Np}, \mathrm{U})$, similar to what we have found in the U-Zr and $\mathrm{Np}-\mathrm{Zr}$ systems and also for $\beta(\mathrm{U})$ of the $\mathrm{Np}-\mathrm{U}$ system above. The large difference in enthalpy of about $0.1 \mathrm{eV} /$ atom between DFT and DFT $+U$ at $\left(\left(U_{\text {eff }}(\mathrm{Np}), U_{\text {eff }}(\mathrm{U})\right)=(0.65\right.$, $1.24) \mathrm{eV}$ ) also suggests the Hubbard $U$ potential in DFT $+U$ has a significant effect for $\zeta(\mathrm{Np}, \mathrm{U})$.

Someone may have concern that these ab initio formation energies for $\zeta(\mathrm{Np}, \mathrm{U})$ even calculated by DFT $+U$ are still positive for all the ordered end members, while a stable compound should typically have a negative formation enthalpy at zero-temperature. The positive values may be in part due to the fact that we are sampling only those end members with several lattice sites grouped together and with each site fully occupied by only one element. The actual $\zeta(\mathrm{Np}, \mathrm{U})$ may have lower calculated enthalpy of formation, which could be found by exploring additional chemical configurations. To estimate the possible scale of stabilization that might be obtained by exploring the whole set of 1024 end members, we take the spread in enthalpies of end members at a same composition (the points on a vertical line in Fig. 3) from the current calculations of the site-grouped end members as a rough guide for the effect of lattice ordering. The spreads are about 0.03 to $0.05 \mathrm{eV} /$ atom, which if subtracted from the current enthalpies, would make the current DFT $+U$ as low as just about zero at U-rich compositions. Another reason for the positive values may be that although $\zeta(\mathrm{Np}, \mathrm{U})$ is stable at room temperature, it is not stable at $0 \mathrm{~K}$, and is stabilized by entropy at finite temperatures. This situation is actually consistent with Kurata's CALPHAD model[9] where $\zeta$ is only stable down to $320 \mathrm{~K}$, as shown in Figure 1. Finally, we note that our ab initio values may be lowered if we explore other $U_{\text {eff }}(\mathrm{Np})$ values in the $0.65-0.90 \mathrm{eV}$ range of single-structure- optimized $U_{\text {eff }}$ for $\mathrm{Np}$ (e.g., the multi-structure optimized $U_{\text {eff }}(\mathrm{Np})$ of $0.90 \mathrm{eV}$ ) and if we include $\mathrm{SOC}$, which typically lowered formation enthalpies 0.02 to $0.03 \mathrm{eV} /$ atom in the $\mathrm{U}-\mathrm{Zr}$ and $\mathrm{Np}-\mathrm{Zr}$ systems, as mentioned above in section 3.

Figure 5 shows the optimized occupations of $\mathrm{Np}$ in the five lattice site groups of $\zeta(\mathrm{Np}, \mathrm{U})$ as a function of composition. We see that standard DFT and DFT $+U$ predict qualitatively and even quantitatively similar lattice site occupation curves despite the significant difference in enthalpy of formation shown above. The most significant difference is only for the occupation curve of lattice site group $\mathrm{V}$ (green), which contains lattice sites 9, and 10. For this group, standard DFT suggests a sharp increase of Np occupation with increasing Np mole fraction, while DFT $+U$ predicts a more gradual increase. This result means that $\mathrm{DFT}+U$ predicts group $\mathrm{V}$ to be more randomly occupied. Another difference is seen in panel a) of Figure 5 that DFT $+U$ predicts two small kinks 
for lattice site group IV (yellow) and V (green) between 5 and 20 at.\%Np. We believe these kinks are numerical artifacts. As seen in Table 4 and Figure 4, the most stable end members from DFT $+U$ (those that overlap with the CEF enthalpy curve) have very similar enthalpies near the U-rich end, making DFT $+U$ more susceptible to some small numerical convergence errors than the standard DFT. As evidence, we show in panel b) of Figure 5 that the two kinks go away if we only lower the enthalpy of the one terminal end member UUUUU by $0.003 \mathrm{eV} /$ atom, which could well be caused by numerical factors like k-point or energy cutoff convergence. Overall, Figure 5 shows that, with increasing mole fraction of $\mathrm{Np}, \mathrm{Np}$ atoms like to occupy lattice sites in group II (orange) first, then group IV (yellow), I (blue), V (green) and finally III (grey), which manifests in Figure 5 as different slopes of the five occupation curves. In particular, group III (grey), which contains only lattice site 4 is almost totally occupied by $\mathrm{U}$ until the overall $\mathrm{Np}$ mole fraction is larger than about 0.8 . This suggests that lattice site 2 is possibly only occupied by $\mathrm{U}$ atoms in the actual $\zeta$ alloy. Lattice sites 9 and 10 in group $\mathrm{V}$ (green) also like less to be occupied by $\mathrm{Np}$ than $\mathrm{U}$, while lattice sites in groups II and IV prefer to be occupied by $\mathrm{Np}$ over U. Lattice site group I, which contains sites 1, 3 and 7 have intermediate $\mathrm{U}$ occupation in a large range of compositions, and thus site 1,3 and 7 possibly do not have strong preference between $\mathrm{Np}$ and $\mathrm{U}$.

Finally, Figure 6 shows occupation of $\mathrm{Np}$ in $\zeta(\mathrm{Np}, \mathrm{U})$ with 60 at. $\% \mathrm{~Np}$ as a function of temperature. At higher temperature, occupation of $\mathrm{Np}$ is lowered in groups II and IV while increased in groups I, III and V. In particular, DFT $+U$ predicts that Np occupation increases strongly at higher temperature in group V. Overall, we expect that the ab initio predicted site occupations may provide some useful information for future thermodynamic description of $\zeta$ phase with a sublattice model, similar to combined $a b$ initio and CALPHAD approach taken in Ref.[37] for the Mo-Re system. For example, group II and IV seem to show very similar composition and temperature dependence of occupation, suggesting they may be further grouped in a four-sublattice model without much loss of interesting physics. Group III and V may also be further grouped in a threesublattice model but some degree of freedom may be lost at high Np content. In a most simplistic two sublattice model, groups I, III and V can be further grouped into one big group because they all have increased occupation of $\mathrm{Np}$ at high temperature in contrast to groups II and IV.

\section{Conclusions}

We performed ab initio calculations of all the solid phases of the Np-U system stable in its current ambient pressure phase diagram. Our work consists of two main parts.

In the first part, we examined if $U_{\text {eff }}(\mathrm{Np})$ and $U_{\text {eff }}(\mathrm{U})$ fitted independently in the $\mathrm{U}-\mathrm{Zr}$ and $\mathrm{Np}-\mathrm{Zr}$ systems may be transferable to the $\mathrm{Np}-\mathrm{U}$ system. We used an existing CALPHAD model as reference, and chose to focus on the terminal $\alpha(\mathrm{Np}), \beta(\mathrm{Np}), \alpha(\mathrm{U})$, $\beta(\mathrm{U})$ and $\mathrm{BCC}$ solution phases $\gamma(\mathrm{Np}, \mathrm{U})$ as the model was examined and found to capture the phase boundary of these phases at least reasonably well based on comparison to available experiential data. We found that DFT $+U$ predicts more or at least comparably accurate enthalpy compared to DFT when using $U_{\text {eff }}(\mathrm{Np})$ and $U_{\text {eff }}(\mathrm{U})$ between 0.65-0.90 $\mathrm{eV}$ and 1-1.5 eV, respectively, which are the ranges of single-structure optimized $U_{\text {eff }}$ 's 
that we determined in our previous studies of the $\mathrm{Np}-\mathrm{Zr}$ and $\mathrm{U}-\mathrm{Zr}$ systems containing either only $\mathrm{Np}$ or only $\mathrm{U}$. This result thus demonstrated the transferability of $U_{\text {eff }}$ 's between U-Zr, Np-Zr and Np-U. Future work would be needed to test if they can be extended to other systems with similar chemical bonding and crystal structures.

In the second part, we focused on the intermediate compound phase $\zeta(\mathrm{Np}, \mathrm{U})$ that is presently poorly understood. Using DFT $+U$ based on the fitted $U_{\text {eff' }}$ 's to calculate formation enthalpies for ordered end members and the compound energy formalism, we predicted $\zeta(\mathrm{Np}, \mathrm{U})$ 's site occupations as function of compositions and temperature. Such detailed knowledge of site occupation and ordering preference is difficult to obtain by fitting CALPHAD to experimental phase boundary data alone, especially for $\zeta(\mathrm{Np}, \mathrm{U})$ as it has a large number of symmetrically distinct sites. These results may be of reference value for future experimental and modeling study of the Np-U system, for example for guiding further grouping of lattice sites based on similarity in the temperature and composition dependences of their occupations in CALPHAD modeling of this complex phase $\zeta(\mathrm{Np}, \mathrm{U})$ using a sublattice model.

\section{Acknowledgement}

This research was funded by the U.S. Department of Energy Office of Nuclear Energy's Nuclear Energy University Programs under contract number 00088978. This work used the resources of the High Performance Computing Center at Idaho National Laboratory, which is supported by the Office of Nuclear Energy of the U.S. Department of Energy under Contract No. DE-AC07-05ID14517. This work also used the Extreme Science and Engineering Discovery Environment (XSEDE), which is supported by National Science Foundation grant number ACI-1053575. We are very grateful for extensive CALPHAD related contributions from Wei Xiong. We also thank Chuan Zhang for various helps and discussions. Finally, we acknowledge CompuTherm, LLC for the use of their Pandat software.

\section{References}

[1] G.L. Hofman, L.C. Walters, T.H. Bauer, Metallic fast reactor fuels, Prog. Nucl. Energy, 31 (1997) 83-110.

[2] P. Hohenberg, W. Kohn, Inhomogeneous Electron Gas, Phys. Rev. B, 136 (1964) 864-871.

[3] W. Kohn, L.J. Sham, Self-Consistent Equations Including Exchange and Correlation Effects, Phys. Rev., 140 (1965) 1133-1138.

[4] S.L. Dudarev, G.A. Botton, S.Y. Savrasov, C.J. Humphreys, A.P. Sutton, Electronenergy-loss spectra and the structural stability of nickel oxide: An LSDA+U study, Phys. Rev. B, 57 (1998) 1505-1509.

[5] W. Xie, W. Xiong, C.A. Marianetti, D. Morgan, Correlation and relativistic effects in U metal and U-Zr alloy: validation of ab initio approaches, Phys. Rev. B, 88 (2013) 235128.

[6] W. Xiong, W. Xie, C. Shen, D. Morgan, Thermodynamic Modeling of the U-Zr System - A Revisit, J. Nucl. Mater., 443 (2013) 331-341. 
[7] W. Xiong, W. Xie, D. Morgan, Thermodynamic evaluation of the Np-Zr system using CALPHAD and ab initio methods, J. Nucl. Mater., 452 (2014) 569-577.

[8] P.G. Mardon, J.H. Pearce, An Investigation of the Neptunium-Uranium Equilibrium Diagram, J. Less-Common Met., 1 (1959) 467-475.

[9] M. Kurata, Thermodynamic database on U-Pu-Zr-Np-Am-Fe alloy system II Evaluation of Np, Am, and Fe containing systems, IOP Conf. Ser.: Mater. Sci. Eng., 9 (2010) 012023.

[10] P. Söderlind, B. Sadigh, V. Lordi, A. Landa, P.E.A. Turchi, Electron correlation and relativity of the $5 f$ electrons in the U-Zr alloy system, J. Nucl. Mater., 444 (2014) 356358.

[11] P. Soderlind, A. Landa, P.E.A. Turchi, Comment on "Correlation and relativistic effects in U metal and U-Zr alloy: Validation of ab initio approaches", Phys. Rev. B, 90 (2014) 157101.

[12] W. Xie, C.A. Marianetti, D. Morgan, Applicability of DFT + U to U metal and U-Zr alloy, arXiv, 1601 (2016) 07959, http://arxiv.org/abs/1601.07959.

[13] W. Xie, C.A. Marianetti, D. Morgan, Response to letter "Electron correlation and relativity of the $5 \mathrm{f}$ electrons in the U-Zr alloy system", J. Nucl. Mater., 476 (2016) 110112.

[14] W. Xie, C.A. Marianetti, D. Morgan, Reply to "Comment on 'Correlation and relativistic effects in U metal and U-Zr alloy: Validation of ab initio approaches" ", Phys. Rev. B, 93 (2016) 157101.

[15] A.C. Lawson, J.A. Goldstone, B. Cort, R.J. Martinez, F.A. Vigil, T.G. Zocco, J.W. Richardson, M.H. Mueller, Structure of $\zeta$-phase plutonium-uranium, Acta Crystallogr. Sect. B, 52 (1996) 32-37.

[16] J.L.C. Daams, J.H.N. Vanvucht, P. Villars, Atomic-Environment Classification of the Cubic Intermetallic Structure Types, J. Alloys Compd., 182 (1992) 1-33.

[17] P. Villars, K. Cenzual, J. Daams, R. Gladyshevskii, O. Shcherban, V. Dubenskyy, N. Melnichenko-Koblyuk, O. Pavlyuk, I. Savysyuk, S. Stoyko, L. Sysa, (U0.4Pu0.6), in: P. Villars, K. Cenzual (Eds.) Structure Types, Springer-Verlag Berlin Heidelberg, 2008.

[18] M. Cococcioni, S. de Gironcoli, Linear response approach to the calculation of the effective interaction parameters in the LDA+U method, Phys. Rev. B, 71 (2005) 035105. [19] A. Zunger, S.H. Wei, L.G. Ferreira, J.E. Bernard, Special Quasirandom Structures, Phys. Rev. Lett., 65 (1990) 353-356.

[20] A. van de Walle, M. Asta, G. Ceder, The Alloy Theoretic Automated Toolkit: A user guide, Calphad, 26 (2002) 539-553.

[21] G. Kresse, J. Hafner, Ab initio Moleculear Dynamics for Liquid Metals, Phys. Rev. B, 47 (1993) 558-561.

[22] G. Kresse, J. Furthmuller, Efficient iterative schemes for ab initio total-energy calculations using a plane-wave basis set, Phys. Rev. B, 54 (1996) 11169-11186.

[23] P.E. Blochl, Projector Augmented-Wave Method, Phys. Rev. B, 50 (1994) 1795317979.

[24] G. Kresse, D. Joubert, From ultrasoft pseudopotentials to the projector augmentedwave method, Phys. Rev. B, 59 (1999) 1758-1775.

[25] D.C. Langreth, J.P. Perdew, Theory of Nonuniform Electronic Systems .1. Analysis of the Gradient Approximation and a Generalization That Works, Phys. Rev. B, 21 (1980) 5469-5493. 
[26] J.P. Perdew, K. Burke, M. Ernzerhof, Generalized gradient approximation made simple, Phys. Rev. Lett., 77 (1996) 3865-3868.

[27] M. Methfessel, A.T. Paxton, High-Precision Sampling for Brillouin-Zone Integration in Metals, Phys. Rev. B, 40 (1989) 3616-3621.

[28] E.R. Davidson, Matrix Eigenvector Methods, in: G.H.F. Diercksen, W. S. (Eds.) Methods in Computational Molecular Physics, D. Reidel Publishing Company, Bad Windsheim, West Germany, 1982, pp. 95-113.

[29] M.P. Teter, M.C. Payne, D.C. Allan, Solution of Schrodinger-Equation for Large Systems, Phys. Rev. B, 40 (1989) 12255-12263.

[30] B. Meredig, A. Thompson, H.A. Hansen, C. Wolverton, A. van de Walle, Method for locating low-energy solutions within DFT plus U, Phys. Rev. B, 82 (2010) 195128.

[31] R. Mathieu, N. Dupin, J.C. Crivello, K. Yaqoob, A. Breidi, J.M. Fiorani, N. David, J.M. Joubert, CALPHAD description of the Mo-Re system focused on the sigma phase modeling, Calphad, 43 (2013) 18-31.

[32] J.L.C. Daams, P. Villars, Atomic environment classification of the tetragonal 'intermetallic' structure types, J. Alloys Compd., 252 (1997) 110-142.

[33] M. Hillert, The compound energy formalism, J Alloy Compd, 320 (2001) 161-176.

[34] S.G. Fries, B. Sundman, Using Re-W sigma-phase first-principles results in the Bragg-Williams approximation to calculate finite-temperature thermodynamic properties, Phys. Rev. B, 66 (2002).

[35] M. Kurata, Thermodynamic database on U-Pu-Zr-Np-Am-Fe alloy system I - Reevaluation of U-Pu-Zr alloy system, IOP Conf. Ser.: Mater. Sci. Eng., 9 (2010) 012022.

[36] P.Y. Chevalier, E. Fischer, B. Cheynet, Progress in the thermodynamic modelling of the O-U-Zr ternary system, Calphad, 28 (2004) 15-40.

[37] Y. Yang, C. Zhang, S.L. Chen, D. Morgan, Y.A. Chang, First-principles calculation aided thermodynamic modeling of the Mo-Re system, Intermetallics, 18 (2010) 574-581. 


\section{Tables}

Table 1. Stable solid phases of the Np-U system, their crystal structures and settings in ab initio calculations.

\begin{tabular}{cccccccc}
\hline Phase & Structure name & $\begin{array}{c}\text { Space } \\
\text { group }\end{array}$ & $\begin{array}{c}\text { Composition } \\
(\mathrm{U} \text { at.\%) }\end{array}$ & $\begin{array}{c}\text { Unit cell size } \\
\text { (atoms/cell) }\end{array}$ & $\begin{array}{c}\text { SQS } \\
\text { used? }\end{array}$ & $\begin{array}{c}k \text {-point } \\
\text { mesh }\end{array}$ & $\begin{array}{c}\text { Expt. } \\
\text { Source }\end{array}$ \\
\hline$\alpha(\mathrm{Np})$ & Orthorhombic_A $\mathrm{A}_{\mathrm{c}}$ & Pnma & 6.3 & 16 & Yes & $8 \times 8 \times 4$ & Ref. [8] \\
$\beta(\mathrm{Np})$ & Tetragonal_A & P4/nmm & 6.3 & 16 & Yes & $6 \times 6 \times 4$ & Ref. [8] \\
& & & 25.0 & & & & \\
$\gamma(\mathrm{Np}, \mathrm{U})$ & Bcc_A2 & $\mathrm{Im} \overline{3} \mathrm{~m}$ & 50.0 & 16 & Yes & $6 \times 6 \times 6$ & Ref. [8] \\
& & & 75.0 & & & & \\
$\beta(\mathrm{U})$ & Tetragonal_A & $\mathrm{P} 6{ }_{2} / \mathrm{mnm}$ & 96.7 & 30 & Yes & $4 \times 4 \times 6$ & Ref. [8] \\
$\alpha(\mathrm{U})$ & Orthorhombic_A20 & $\mathrm{Cmcm}$ & 93.8 & 16 & Yes & $8 \times 8 \times 4$ & Ref. [8] \\
$\zeta(\mathrm{Np}, \mathrm{U})$ & Tetragonal_? & $\mathrm{R} \overline{3} \mathrm{~m}$ & $0-100$ & 58 & No & $2 \times 2 \times 2$ & Ref. [15] \\
\hline
\end{tabular}


Table 2. Structural information for lattice sites of $\zeta(\mathrm{Np}, \mathrm{U})$. The space group $\mathrm{R} \overline{3} \mathrm{~m}$ (no.166) and lattice site coordinates were determined for $\zeta$ phase in the Pu-U system in Ref.[15]. $\zeta(\mathrm{Np}, \mathrm{U})$ in the $\mathrm{Np}-\mathrm{U}$ system was suggested to be isomorphous with $\zeta(\mathrm{Pu}, \mathrm{U})$ the $\mathrm{Pu}-\mathrm{U}$ system[8]. Coordination number is here defined as number of neighboring atoms within $3.7 \AA$ (see the body of text) of the center atom and the values are quoted from Ref.[15]. Atomic environment are quoted from Ref.[17].

\begin{tabular}{ccccc}
\hline $\begin{array}{c}\text { Lattice } \\
\text { site }\end{array}$ & $\begin{array}{c}\text { Wyckoff } \\
\text { label }\end{array}$ & $\begin{array}{c}\text { Site } \\
\text { symmetry }\end{array}$ & $\begin{array}{c}\text { Coordination } \\
\text { number }\end{array}$ & Atomic environment \\
\hline 1 & $36 i$ & 1 & 14 & 14-vertex Frank-Kasper \\
2 & $18 h$ &.$m$ & 13 & pseudo Frank-Kasper \\
3 & $18 h$ &.$m$ & 14 & 14-vertex Frank-Kasper \\
4 & $18 h$ &.$m$ & 15 & 15-vertex Frank-Kasper \\
5 & $18 h$ &.$m$ & 13 & pseudo Frank-Kasper \\
6 & $18 h$ &.$m$ & 13 & pseudo Frank-Kasper \\
7 & $18 h$ &.$m$ & 14 & 14-vertex Frank-Kasper \\
8 & $18 f$ & .2 & 12 & icosahedron \\
9 & $6 c$ & $3 m$ & 16 & 16-vertex Frank-Kasper \\
10 & $6 c$ & $3 m$ & 16 & 16-vertex Frank-Kasper \\
\hline
\end{tabular}


Table 3. Groups of lattice sites of $\zeta(\mathrm{Np}, \mathrm{U})$ in $a b$ initio calculations according to coordination number/atomic environment. $f_{\mathrm{g}}$ is the average fraction of same-group neighbor atoms of a site, which can be calculated as $f_{g}=a_{s g}^{s} f_{g}^{s}$ where $f_{g}^{s}$ is the fraction of neighboring atoms that are in the same group $g$ with the center atom on lattice site $s$, and $a_{g}^{s}$ is the fraction of lattice site $s$ in group $g$.

\begin{tabular}{cccccc}
\hline Group & Fraction $a^{g}$ & Lattice sites & $\begin{array}{c}\text { Coordination } \\
\text { number }\end{array}$ & Atomic environment & $f_{\mathrm{g}}$ \\
\hline I & $24 / 58$ & $1,3,7$ & 14 & 14-vertex Frank-Kasper & 0.39 \\
II & $18 / 58$ & $2,5,6$ & 13 & pseudo Frank-Kasper & 0.21 \\
III & $6 / 58$ & 4 & 15 & 15-vertex Frank-Kasper & 0 \\
IV & $6 / 58$ & 8 & 12 & icosahedron & 0.17 \\
V & $4 / 58$ & 9,10 & 16 & 16-vertex Frank-Kasper & 0.06 \\
\hline
\end{tabular}


Table 4. Enthalpy of formation for $\zeta(\mathrm{Np}, \mathrm{U})$. Ab initio values both calculated for the 32 ordered end members and predicted by $\mathrm{CEF}$ at the 20 compositions where ordered end members exist are provided. CALPHAD enthalpies at $300 \mathrm{~K}$ are from Kurata[9].

\begin{tabular}{|c|c|c|c|c|c|c|}
\hline \multirow{2}{*}{$x(\mathrm{~Np})$} & \multirow{2}{*}{ End member } & \multicolumn{2}{|c|}{ DFT $(0 \mathrm{~K})$} & \multicolumn{2}{|c|}{$\mathrm{DFT}+U(0 \mathrm{~K})$} & \multirow{2}{*}{$\begin{array}{l}\text { CALPHAD } \\
(300 \mathrm{~K})\end{array}$} \\
\hline & & End member & CEF & End member & CEF & \\
\hline 1.00 & NpNpNpNpNp & 0.276 & 0.276 & 0.136 & 0.136 & 0.052 \\
\hline 0.93 & NpNpNpNpU & 0.260 & 0.254 & 0.123 & 0.116 & 0.044 \\
\hline \multirow{2}{*}{0.90} & NpNpNpUNp & 0.272 & \multirow{2}{*}{0.244} & 0.133 & \multirow{2}{*}{0.107} & \multirow{2}{*}{0.039} \\
\hline & NpNpUNpNp & 0.243 & & 0.106 & & \\
\hline \multirow{2}{*}{0.83} & NpNpNpUU & 0.247 & \multirow{2}{*}{0.218} & 0.117 & \multirow{2}{*}{0.097} & \multirow{2}{*}{0.029} \\
\hline & NpNpUNpU & 0.217 & & 0.097 & & \\
\hline 0.79 & NpNpUUNp & 0.231 & 0.210 & 0.103 & 0.092 & 0.023 \\
\hline 0.72 & NpNpUUU & 0.202 & 0.197 & 0.086 & 0.084 & 0.013 \\
\hline 0.69 & NpUNpNpNp & 0.224 & 0.192 & 0.098 & 0.080 & 0.009 \\
\hline 0.62 & NpUNpNpU & 0.210 & 0.105 & 0.098 & 0.037 & 0.003 \\
\hline \multirow{3}{*}{0.59} & NpUUNpNp & 0.202 & \multirow{3}{*}{0.173} & 0.088 & \multirow{3}{*}{0.068} & \multirow{3}{*}{0.000} \\
\hline & NpUNpUNp & 0.218 & & 0.103 & & \\
\hline & UNpNpNpNp & 0.202 & & 0.087 & & \\
\hline \multirow{3}{*}{0.52} & NpUUNpU & 0.190 & \multirow{3}{*}{0.160} & 0.081 & \multirow{3}{*}{0.059} & \multirow{3}{*}{-0.003} \\
\hline & UNpNpNpU & 0.188 & & 0.080 & & \\
\hline & NpUNpUU & 0.200 & & 0.092 & & \\
\hline \multirow{3}{*}{0.48} & NpUUUNp & 0.192 & \multirow{3}{*}{0.153} & 0.088 & \multirow{3}{*}{0.055} & \multirow{3}{*}{-0.003} \\
\hline & UNpNpUNp & 0.206 & & 0.080 & & \\
\hline & UNpUNpNp & 0.168 & & 0.058 & & \\
\hline \multirow{3}{*}{0.41} & NpUUUU & 0.171 & \multirow{3}{*}{0.140} & 0.084 & \multirow{3}{*}{0.046} & \multirow{3}{*}{-0.002} \\
\hline & UNpNpUU & 0.179 & & 0.069 & & \\
\hline & UNpUNpU & 0.141 & & 0.047 & & \\
\hline 0.38 & UNpUUNp & 0.142 & 0.136 & 0.058 & 0.044 & -0.001 \\
\hline 0.31 & UNpUUU & 0.128 & 0.128 & 0.039 & 0.039 & 0.003 \\
\hline 0.28 & UUNpNpNp & 0.177 & 0.124 & 0.067 & 0.039 & 0.005 \\
\hline 0.21 & UUNpNpU & 0.153 & 0.116 & 0.061 & 0.039 & 0.009 \\
\hline 017 & UUNpUNp & 0.166 & 0111 & 0.057 & 0030 & 0011 \\
\hline 0.17 & UUUNpNp & 0.128 & 0.111 & 0.043 & 0.059 & 0.011 \\
\hline 0.10 & UUUNpU & 0.106 & 0103 & 0.037 & 0037 & 0011 \\
\hline & UUNpUU & 0.137 & 0.105 & 0.059 & 0.031 & 0.011 \\
\hline 0.07 & UUUUNp & 0.117 & 0.099 & 0.038 & 0.037 & 0.010 \\
\hline 0.00 & UUUUU & 0.091 & 0.091 & 0.038 & 0.038 & 0.004 \\
\hline
\end{tabular}




\section{Figures}

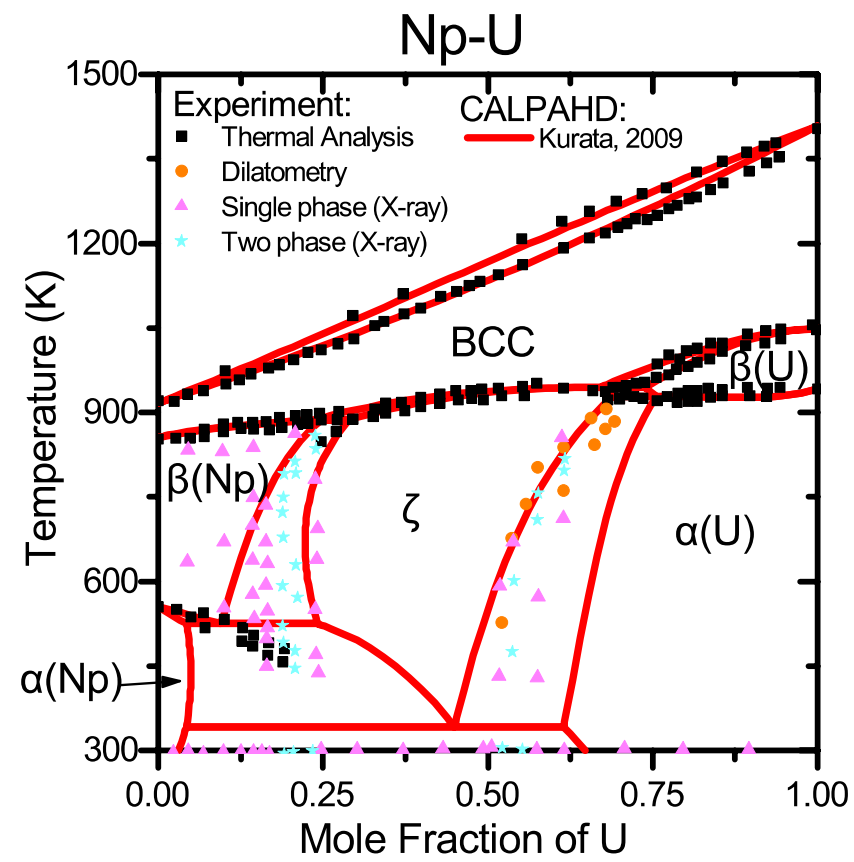

Figure 1. Phase diagram of the Np-U system from the CALPHAD model of Kurata[9] compared to the experimental data of Mardon and Pearce[8]. 

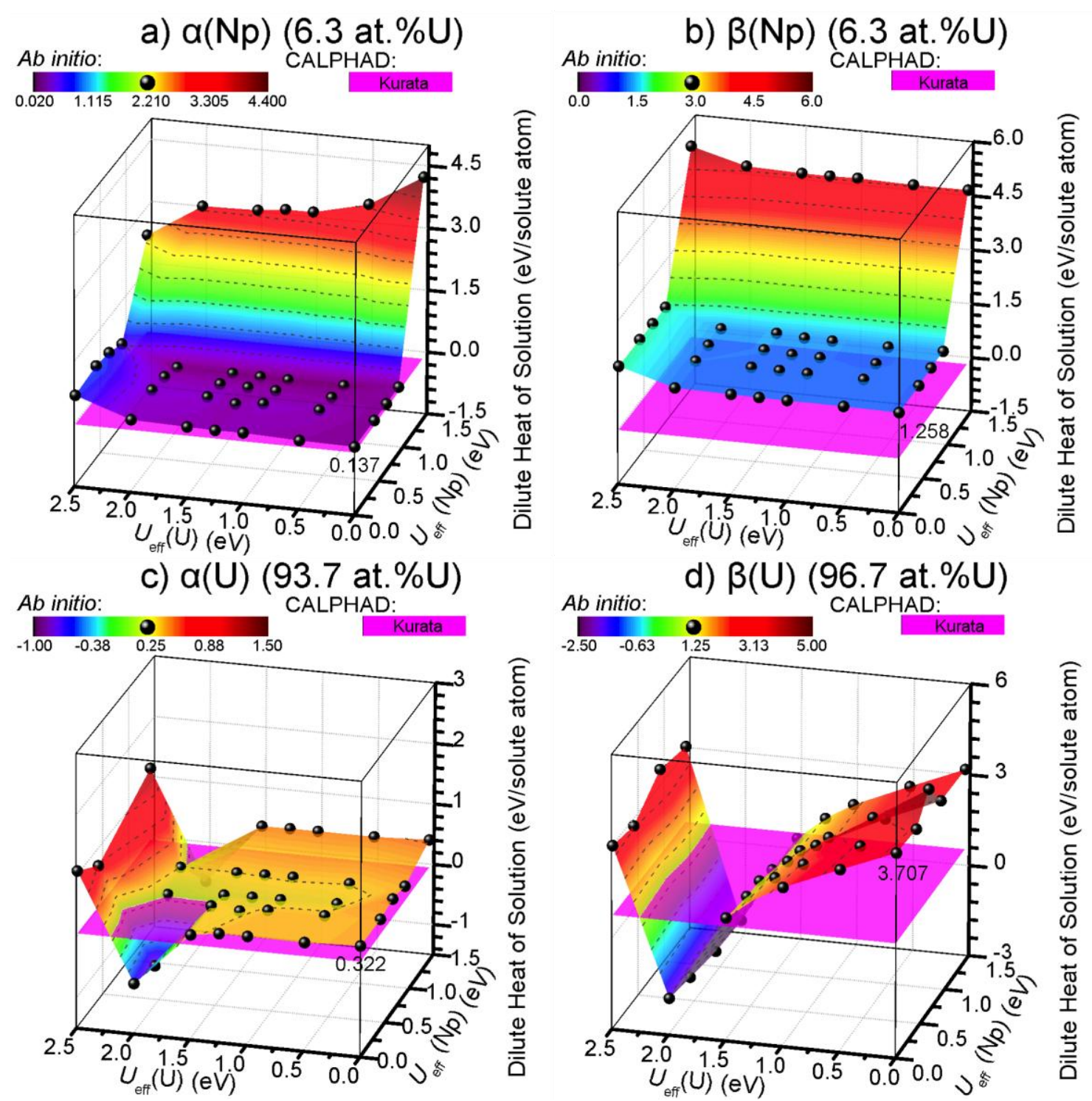

Figure 2. Dilute heat of solution for a) $\alpha(\mathrm{Np})$ (6.3 at.\%U), b) $\beta(\mathrm{Np})(6.3$ at.\%U), c) $\alpha(\mathrm{U})(93.7$ at.\% $\mathrm{U})$, and d) $\beta(\mathrm{U})(96.7$ at.\% $\%)$ from $a b$ initio at different $U_{\text {eff }}(\mathrm{Np})$ and $U_{\text {eff }}(\mathrm{U})$ compared with those from the CALPHAD models of Kurata (magenta)[9]. Only the points marked with black balls are actually calculated data, and the surfaces connecting them are from spline interpolation as guides to the eyes. 


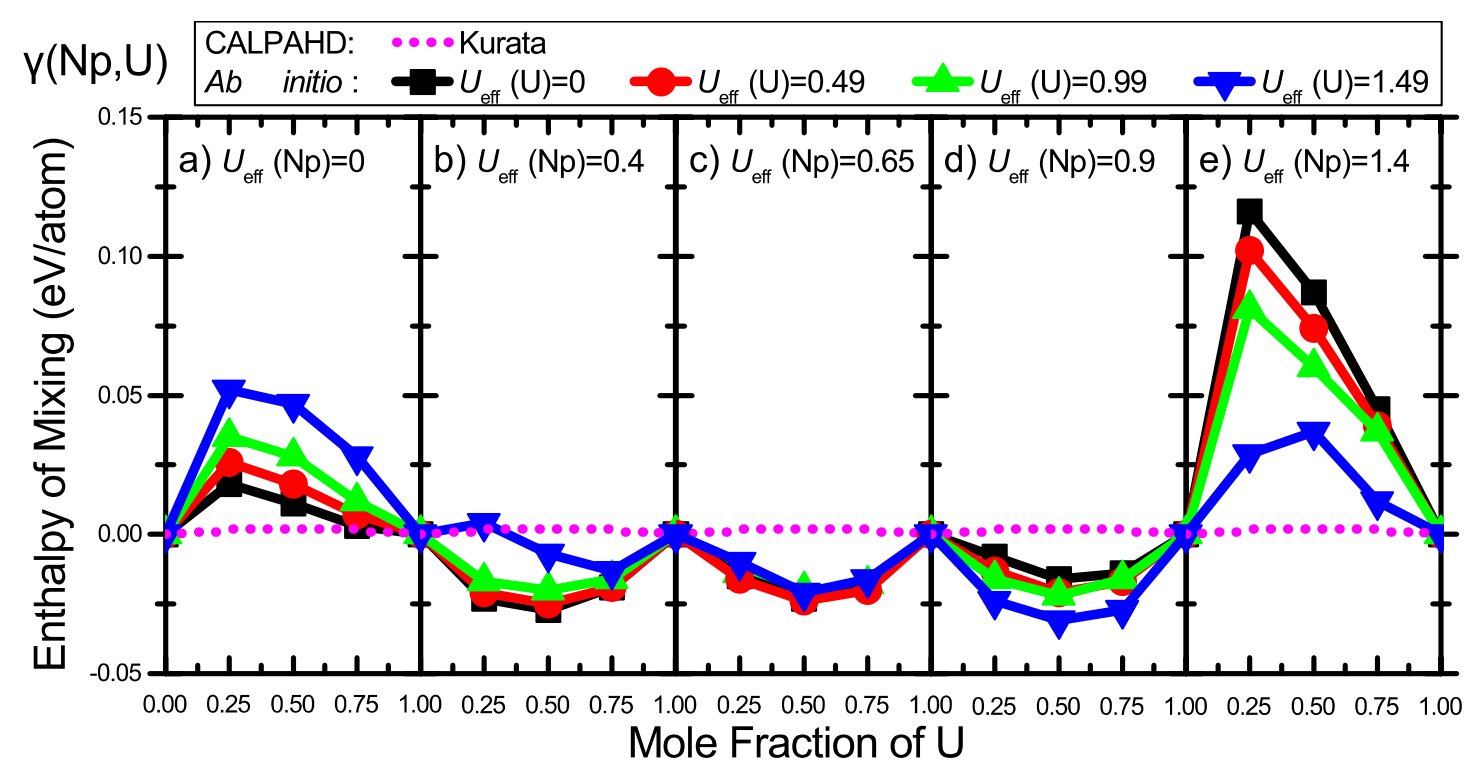

Figure 3. Enthalpy of mixing for $\gamma(\mathrm{Np}, \mathrm{U})$ from $a b$ initio compared with those from the CALPHAD models of Kurata (magenta)[9]. The results from $\left.U_{\text {eff }}(N p)=a\right) 0$, b) 0.4, c) 0.65, d) 0.9 , e) $1.40 \mathrm{eV}$ are shown in the respective panel. In all panels the results from $U_{\text {eff }}(\mathrm{U})=0$ (black), 0.49 (red), 0.99 (green) and 1.49 (blue) $\mathrm{eV}$ are shown as different curves. Only the points marked with symbols are actually calculated data, and the lines connecting them are from spline interpolation as guides to the eyes. 


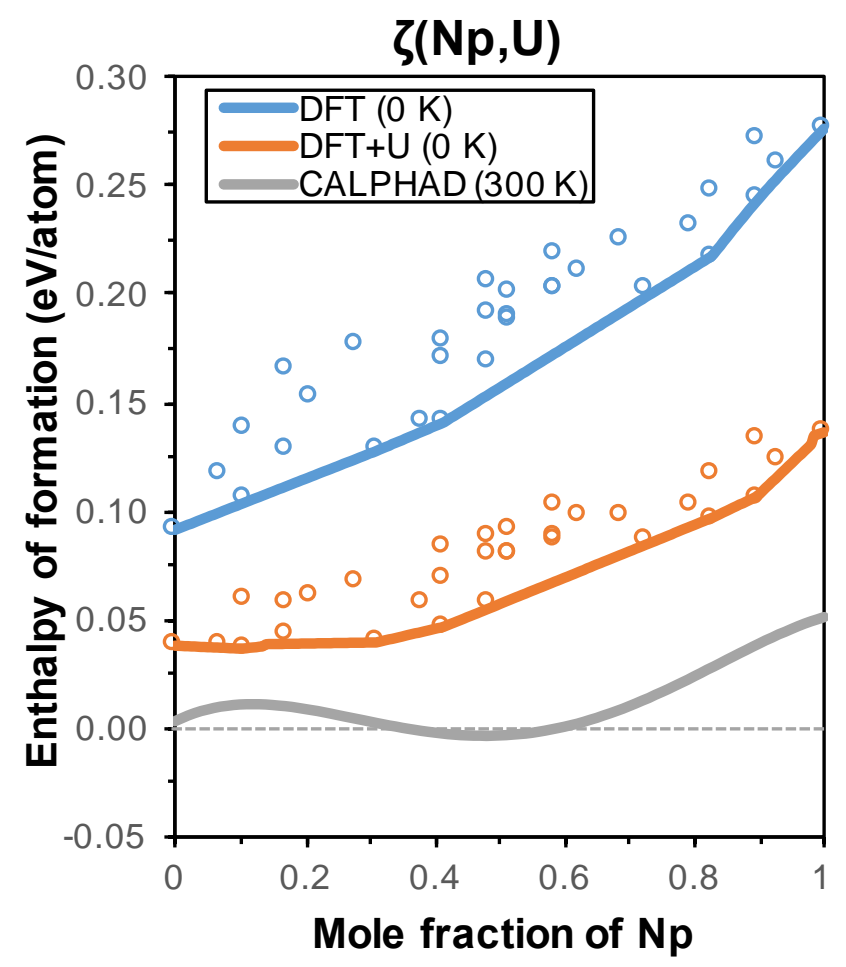

Figure 4. Enthalpy of formation for $\zeta(\mathrm{Np}, \mathrm{U})$ calculated by DFT (blue) and DFT $+U$ (orange) at $\left(U_{\text {eff }}(\mathrm{Np}), U_{\text {eff }}(\mathrm{U})\right)=(0.65,1.24) \mathrm{eV}$ at $0 \mathrm{~K}$ compared to those from Kurata's CALPHAD model[9] (grey) 54at $300 \mathrm{~K}$. For DFT and DFT $+U$, open circle symbol and solid line denote calculated value of specific end members and CEF values, respectively. 

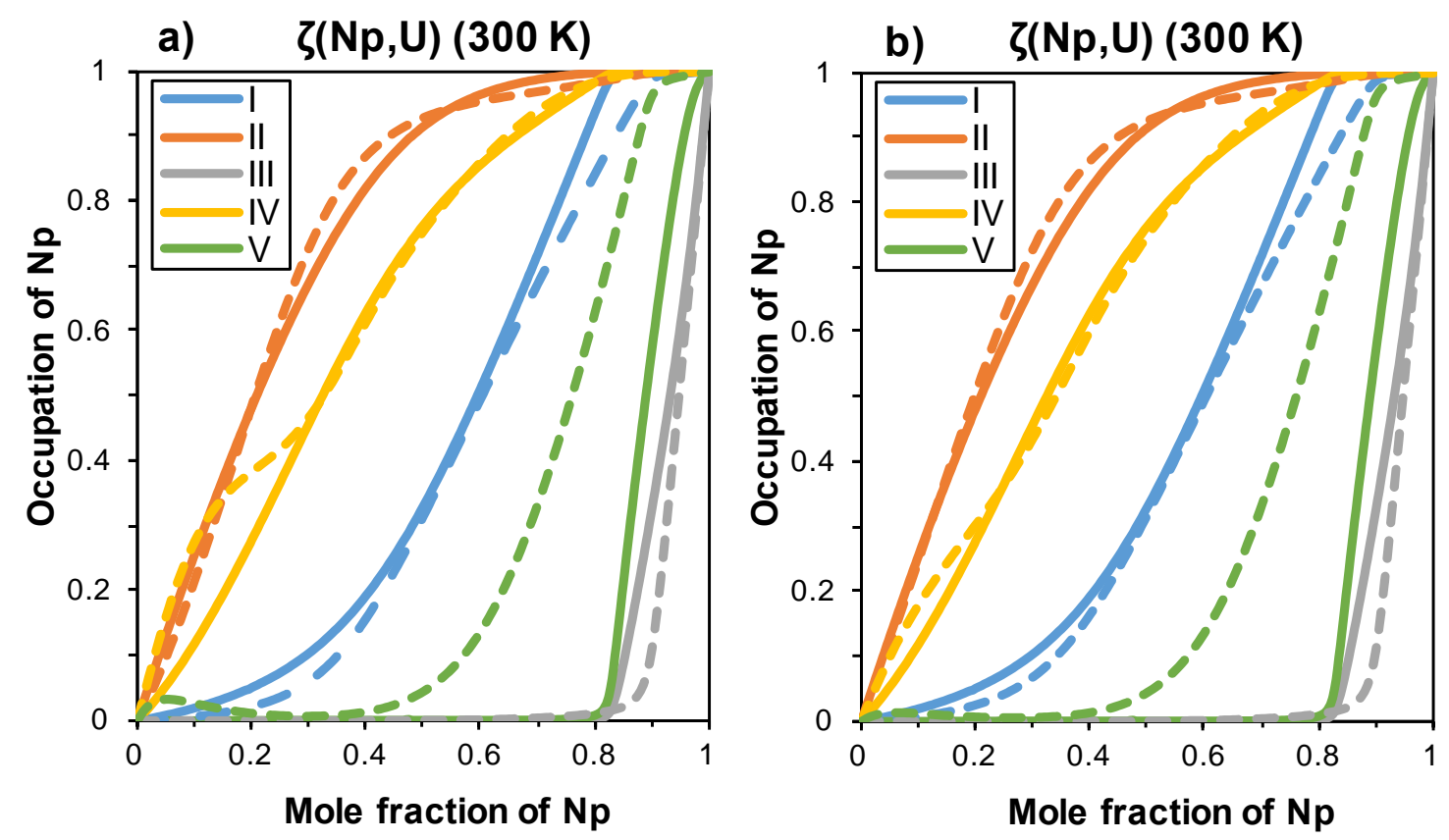

Figure 5. Occupation of $\mathrm{Np}$ in the five lattice site groups I-V of $\zeta(\mathrm{Np}, \mathrm{U})$ as a function of composition at $300 \mathrm{~K}$ based on end member enthalpies calculated by DFT (solid) and DFT $+U$ $($ dash $)$ at $\left(U_{\text {eff }}(\mathrm{Np}), U_{\text {eff }}(\mathrm{U})\right)=(0.65,1.24) \mathrm{eV}$. DFT $+U$ Enthalpy of formation for end member UUUUU in a) is actually calculated while in b) is adjusted $0.003 \mathrm{eV} /$ atom smaller with those for all others kept unchanged. 


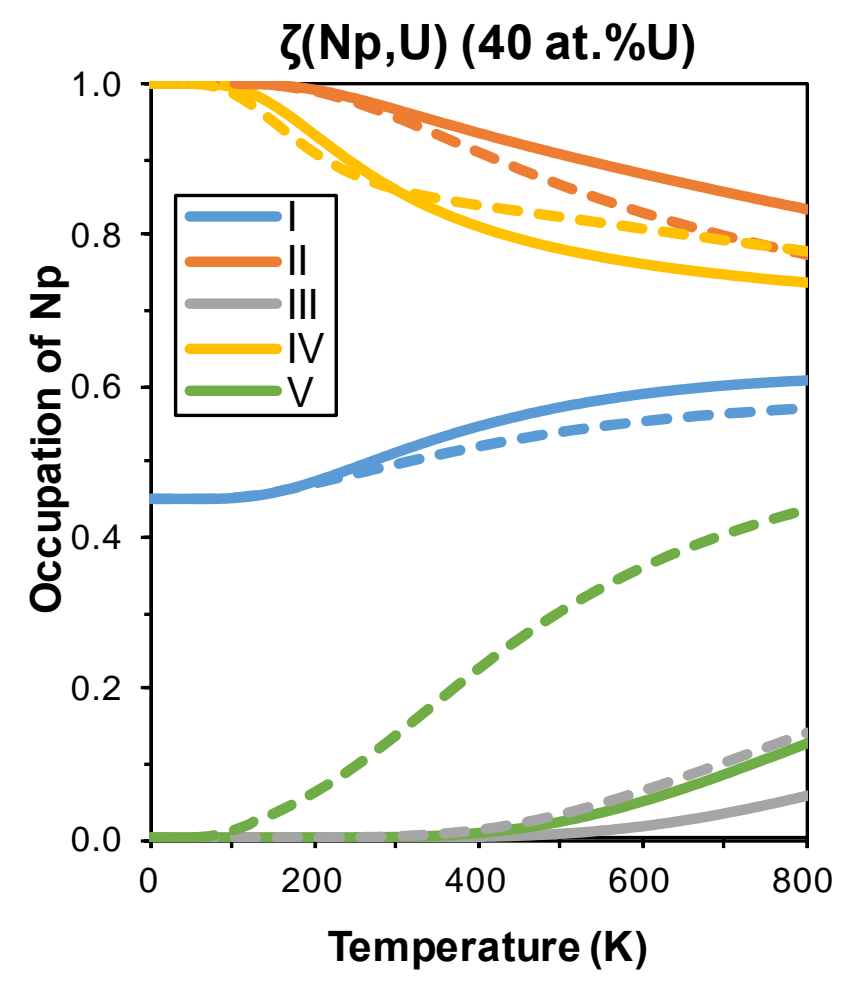

Figure 6. Occupation of $\mathrm{Np}$ in the five lattice site groups $\mathrm{I}-\mathrm{V}$ of $\zeta(\mathrm{Np}, \mathrm{U})$ at 60 at. $\% \mathrm{~Np}$ as a function of temperature based on end member enthalpies calculated by DFT (solid) and DFT $+U$ $($ dash $)$ at $\left(U_{\text {eff }}(\mathrm{Np}), U_{\text {eff }}(\mathrm{U})\right)=(0.65,1.24) \mathrm{eV}$. 